\title{
Comparison of Aerosolized Hydrogen Peroxide Fogging with a Conventional Disinfection Product for a Dental Surgery
}

\author{
Marc Sher ${ }^{1}$, Riaan Mulder ${ }^{2}$
}

\begin{abstract}
Aim and objective: The study aimed to determine the efficacy of adding a 12\% hydrogen peroxide dry hydrogen peroxide vapor fogger system as an additional layer of infection control in a dental surgery.

Materials and methods: A total number of agar plates from the five locations were used during the treatment of the 22 patients $(n=440)$. During the treatment of each patient, four agar plates $(n=4)$ were used per location [location 1: X-ray, location 2: the dental arm, location 3: left side desk, location 4: under the foot of the dental chair, location 5: right side desk and ( $n=20$ for the five locations per patient)]. The control agar plates were incubated after the treatment of the patient was completed period. The test agar plate groups were sprayed with a $70 \%$ isopropanol surface disinfectant, or received exposure to an automated $12 \%$ hydrogen peroxide fog, or a $70 \%$ isopropanol surface disinfectant spray immediately followed by exposure to the automated $12 \%$ hydrogen peroxide.

Results: One-way ANOVA and Scheffé's method identified significant differences $(p<0.01)$. Between the control agar plates and the three disinfection methods used a significantly lower colony count was established for colonies recorded in the surgery assessed as a whole, the X-ray unit, and the right side desk.

Conclusion: The disinfection of dental surgery requires sufficient time as it not only includes the working surfaces but also various inanimate objects. Surface disinfectant spray followed by hydrogen peroxide decontamination has potential for dental surgery, as the colony-forming units have been reduced further compared to spray alone and even just fog alone for all the various areas of the dental surgery that was assessed. Clinical significance: The infection control protocols with hydrogen peroxide vapor would ensure the maximum efficacy of the disinfection protocols.
\end{abstract}

Keywords: Colony-forming units, Dental surgery, Fogger, Hydrogen peroxide vapor, Surface disinfectant.

The Journal of Contemporary Dental Practice (2020): 10.5005/jp-journals-10024-2983

\section{INTRODUCTION}

The survival and cross-contamination of nosocomial infection in hospitals had been established. ${ }^{1}$ It is known that the dental environment is exposed to various infectious agents originating from the oral cavities of patients. ${ }^{2,3}$ The aerosol generated in dental surgery has been termed bioaerosol or microbial aerosol. ${ }^{4}$ It had been suggested the whole dental surgery is likely to be contaminated from the generated droplets and aerosols from infected patients. ${ }^{5}$ Inanimate surfaces remain a problematic component of surface disinfection. ${ }^{6}$ Additionally, the COVID-19 pandemic of 2019/2020 had changed the perceptions of many dental practitioners in terms of the minimum required PPE to treat patients, ${ }^{7}$ although the basic principles had been known. ${ }^{8}$

The production of aerosol from ultrasonic devices ${ }^{9}$ and air-powered turbines ${ }^{10}$ generate aerosol and are widely used in daily dental practice. It had been determined that during extractions, air turbines with water spray, and ultrasonic procedures the microorganism level in the dental surgery returned to normal after $10-30$ minutes. ${ }^{11}$ Therefore, the risk of dental practice becoming a nexus for disease transmission due to the high volume of aerosol production on a daily basis ${ }^{12}$ is a growing concern. In the dental environment, alcohol-containing products have become popular as a surface disinfectant. Alcoholcontaining disinfectant formulations usually contain only aqueous solutions of isopropyl or ethyl alcohol at a percentage between 60 and $90 \%$. These products do not require registration at the Environmental Protection Agency in the United States. ${ }^{13}$ Variations in the names given to the three main types of alcohol used most commonly in healthcare include (1) ethyl alcohol or ethanol; (2)
${ }^{1}$ MarcSher Dental, Department of Dentistry, Sea Point Medical Centre, Cape Town, South Africa

${ }^{2}$ Restorative Dentistry, Department of Prosthetics, University of the Western Cape, Cape Town, South Africa

Corresponding Author: Riaan Mulder, Restorative Dentistry, Department of Prosthetics, University of the Western Cape, Cape Town, South Africa, Phone: +27 (0) 219373156, e-mail: rmulder@uwc.ac.za

How to cite this article: Sher M, Mulder R. Comparison of Aerosolized Hydrogen Peroxide Fogging with a Conventional Disinfection Product for a Dental Surgery. J Contemp Dent Pract 2020;21(12):1307-1311.

Source of support: Nil

Conflict of interest: None

isopropyl alcohol, isopropanol, 2-propanol, or propan-2-ol; and (3) n-propanol, 1-propanol, or propan-1-ol. ${ }^{13}$ The CDC classifies the alcohol-containing disinfectants as intermediate-level germicides. ${ }^{6}$ Hence, the ideal would be to clean the surface before disinfecting it with the $>70 \%$ alcohol spray. ${ }^{14}$ The surface disinfection of the $70 \%$ alcohol is widely used as a relatively quick and easy method for cleaning the non-critical surfaces in the practice where aerosol could settle. The two popular techniques in dentistry are spray or the spray-wipe-spray between patients, but one author postulating a valid question to the efficacy of safe practice. ${ }^{15}$ Additionally, $>70 \%$ of alcohols are popular in dentistry is that they are fungicidal and virucidal against viruses that are of concern to the oral healthcare workers who have lipid envelopes, e.g., herpes virus I, II, V-XIII, ${ }^{15}$ human immunodeficiency virus-1, hepatitis $\mathrm{B}^{16}$, and $\mathrm{C}^{17}$ viruses. The alcohol acts by denaturing 
the proteins of the microorganism ${ }^{6}$ and then destroying the cytoplasm. ${ }^{15}$

In the present study, the purpose was to assess the efficacy of adding a layer of infection control in a dental surgery. The use of a $12 \%$ dry vaporized hydrogen peroxide (VHP) fogger system with and without the layers of infection control was compared.

\section{Materials and Methods}

\section{Ethical Considerations}

The information collected from the bacteria that settled on agar plates was considered to be part of the compilation of a clinical data set. The authors further used a patient consent form that was completed, signed, and saved securely. A statement confirming consent from the patients to compile the data set and to publish has been obtained. No procedures impacting the treatment of the patients were performed with any deviation of routine dentistry, but ethical standards by following the 1964 Helsinki declaration and its later amendments or comparable ethical standards were observed. Agar plates were anonymized in terms of patient details, therefore only the location and treatment were recorded on the agar plates. Agar plates were destroyed following biohazard guidelines. No microbial DNA or RNA is extracted from the agar plates. Patients could at any stage decide to exclude their data set of agar plate results without any recourse or influence on their treatment. Patients were not incentivized for permission to place the agar plates.

\section{Sample Size}

The sample size was calculated from the data collected in Table 1 for the control of the whole surgery in relation to the surface disinfectant results for the whole surgery; originating from 220 agar plates used for the control plus the surface disinfectant component of the study. The sample size was confirmed to be sufficient with a Mann-Whitney test calculator with the values based on the median value of the whole surgery [19.3 colony-forming unit (CFU)] in relation to the surface disinfectant median (8.5 CFU) with a population standard deviation of 25.1 (Table 1). The confidence level and power of the test was $95 \%$. The sample size calculation to compare the aforementioned medians was determined to be a minimum of 161 agar plates.

In this study, the total number of agar plates from the five locations were used during the treatment of the 22 patients ( $n=$ $440)$. During the treatment of each patient, four agar plates $(n=4)$ were used per location [location 1:X-ray, location 2: the dental arm,

Table 1: Mean values and the standard deviation for the technique used in the various locations of the surgery

\begin{tabular}{lllll}
\hline $\begin{array}{l}\text { Area of } \\
\text { surgery }\end{array}$ & Control & $\begin{array}{l}\text { Alcohol } \\
\text { spray }(S P)\end{array}$ & SP + FG & FOG $(F G)$ \\
\hline $\begin{array}{l}\text { Surgery as a } \\
\text { whole }\end{array}$ & $19.3( \pm 35.6)$ & $8.5( \pm 14.6)^{*}$ & $2.8( \pm 3.7)^{*}$ & $4.8( \pm 6.5)^{*}$ \\
$\begin{array}{l}\text { X-ray } \\
\text { Right side }\end{array}$ & $13.1( \pm 7.5)$ & $4.9( \pm 4.3)^{*}$ & $2.8( \pm 4.5)^{*}$ & $4.2( \pm 5.0)^{*}$ \\
desk & $14.9( \pm 13.3)$ & $5.3( \pm 6.0)^{*}$ & $1.8( \pm 2.8)^{*}$ & $4.3( \pm 4.9)^{*}$ \\
$\begin{array}{l}\text { Under the } \\
\text { dental chair }\end{array}$ & $15.8( \pm 11.2)$ & $13.9( \pm 20.4)$ & $3.1( \pm 3.6)$ & $6.8( \pm 10.3)$ \\
$\begin{array}{l}\text { Dental arm } \\
\text { Left side desk }\end{array}$ & $27.3( \pm 46.2)$ & $12.8( \pm 22.8)$ & $3.4( \pm 3.3)^{*}$ & $6.2( \pm 7.5)$ \\
\hline
\end{tabular}

*Indicate a significant difference with the control location 3: left side desk, location 4: under the foot of the dental chair, location 5: right side desk and ( $n=20$ for the five locations)]. The four plates per location were grouped in a circle with the edges $5 \mathrm{~mm}$ apart. At each location, one plate served as the control and was closed immediately after the procedure with no treatment exposure. The other three plates at each location would receive the disinfectant and/or the fogging at the same time at the end of the session.

The fogging time of the dental surgery allowed for three patients to be treated per day, with the treatment period standardized at 30 minutes. It took eight consecutive days to treat 22 patients. The procedures were limited to restorations placement with a fast handpiece or ultrasonic scaler prophylaxis treatment.

\section{Surface Disinfectant}

The plates were sprayed with a $70 \%$ surface disinfectant (SP). A metered-dose of $1 \mathrm{~mL}$ was sprayed $15 \mathrm{~cm}$ away from the agar plate. The agar plate cover was replaced and incubated. The plates receiving the alcohol spray followed by fogging were sprayed immediately before fogging (SP + FG).

\section{Hydrogen Peroxide Fogging}

After the placement of all the agar plates collected for the day, the lids were removed, the dental surgery was exited. The air conditioner is $2 \mathrm{~m}$ from the ground and the airflow direction is set toward location 4. The unit turned on at $440 \mathrm{~L} /$ minute at a temperature of $23^{\circ} \mathrm{C}$. The fogger delivered the hydrogen peroxide disinfectant into the dental surgery. Using the automated hydrogen peroxide vapor fogger (Nocospray: Oxypharm, Champingy-surMarne, France) and the 12\% hydrogen peroxide liquid (Nocolyse: Oxypharm, Champingy-sur-Marne, France), the dental surgery was fogged (FG). The location of the fogger was in one corner of the dental surgery (Fig. 1). The machine was equipped with a Diffuser nozzle, venturi design at $3.4 \times 2.4 \mathrm{~cm}$ and set at the following settings in accordance with the manufacturer's instructions: fogging at $16.6 \mathrm{~mL} /$ minute until $75 \mathrm{~mL} / \mathrm{m}^{3}$, dwell time 120 minutes. Then, the ventilation was resumed, and aeration was allowed for 20 minutes to remove the fogged disinfectant before the agar plate lids were replaced.

\section{Experimental Conditions and Agar Plates}

The agar plates (TSA LTHth-ICR: Merck Life Science GmbH, Eppelheim, Germany, Batch 140477) were placed in the various locations of the dental surgery illustrated in Figure 1. The dental surgery room size was $5 \mathrm{~L} \times 5 \mathrm{~W} \times 3 \mathrm{H}$ meters. The areas included the five locations. The height of the surfaces from the floor was $1.2 \mathrm{~m}$ for the left and right side desk, $1 \mathrm{~m}$ for the dental arm, $1.8 \mathrm{~m}$ for the $X$-ray, and on the floor under the foot of the dental chair. The covers were removed at the commencement of the dental procedure and replaced at the end of the day.

\section{Method of Culture}

After the agar plates receiving the selected treatment, they were incubated for 48 hours at $37^{\circ} \mathrm{C}$. The bacterial and fungal colonies were counted with a colony counter. No viral assessment was completed on the agar plates.

\section{Statistical Analysis}

The statistical analysis was completed with one-way ANOVA and the post hoc Tukey HSD test. Scheffé's method was used to identify the significant differences identified by the Tukey HSD test [Statistical 


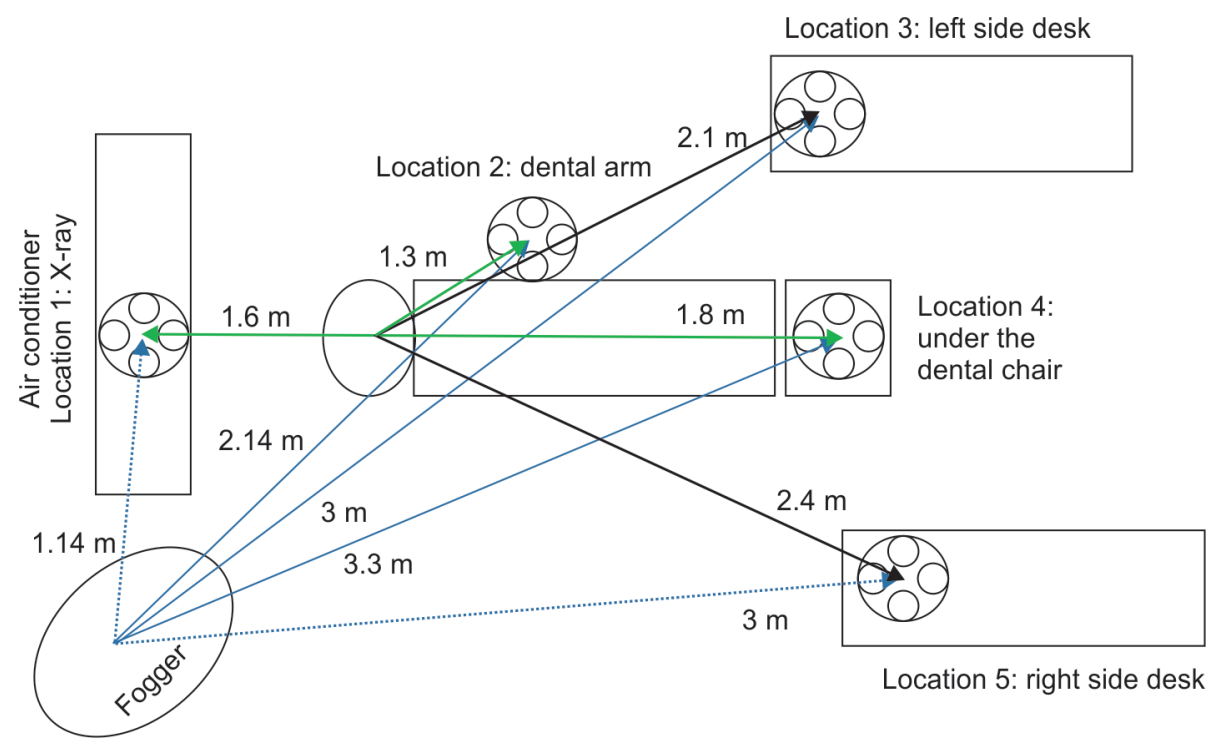

Fig. 1: Location of the various surfaces in the surgery where agar plates were placed in relation to the orientation of the fogger

analysis with R Core Team (2013); (R: A language and environment for statistical computing. R Foundation for Statistical Computing, Vienna, Austria)].

\section{Results}

The $p$ value corresponding to the $F$-statistic for the one-way analysis of variance (ANOVA) is lower than 0.05 for the surgery as a whole. This result indicate that one or more treatments are significantly different. Scheffé's method was used to identify the significant differences.

The analysis for the colonies counted on the agar plates for the surgery as a whole, as well as the individual areas indicated that some disinfection methods were significantly different from the number of colonies counted on the control agar plates $(p<0.01)$. The Scheffé's test indicated that all the disinfection methods had a significantly lower colony count than the control agar plates for the surgery assessed as a whole, the X-ray unit, and the right side desk.

The assessment of significance between the three different disinfection methods indicated that there was no significant difference between the three methods (SP, SP + FG, and FG) for the colonies counted when all the areas of the surgery were assessed as a whole. The same result was observed for the specific areas where agar plates were placed: X-ray unit, right side desk, under the chair, dental arm, and left side desk (Table 1). Concerning the dental arm the only significant difference $(p<0.01)$ between the numbers of colonies counted for the control agar plates and the spray with fog colonies on the agar plates. None of the other two disinfection methods had a significant difference with the control.

\section{Discussion}

In light of the recent COVID-19 pandemic, there has been a large focus in dentistry toward the use of air-decontamination as well as large scale decontamination of these enclosed spaces with various fogger systems. To this extent, fogging had been adopted by some practitioners, although the efficacy toward COVID-19 and the general efficacy in the dental environment is yet to be established. Disinfectant properties and uses of ethyl alcohol or isopropyl alcohol (60-90\%) (v/v), when used as aqueous solutions without other ingredients have been studied in the literature. ${ }^{16-20}$
Ethyl alcohol is more effective in disinfection than isopropanol. Ethyl alcohol is effective against vegetative bacteria. ${ }^{21}$ Subsequent in vitro suspension tests revealed that $60-90 \%$ ethyl alcohol concentrations were the most effective within 1 minute, against three healthcare pathogens ${ }^{22}$ in vitro studies were performed with bacteria dried in a thin smear layer and killed within 30 seconds by $70 \%$ isopropanol. ${ }^{23}$ For this study, the agar plates located in the various locations would get inoculated with microorganisms that settle on the specific agar plates during the duration of the dental procedures (Fig. 1). The treatments performed in dental surgery create aerosol. Aerosols are liquid and solid particles $<50 \mu \mathrm{m}$ in diameter, suspended in air, which are not always visible to the naked eye. Splatter is usually described as a mixture of air, water, and/or solid substances, water droplets in splatter are from $>50 \mu \mathrm{m}$ to several millimeters in diameter and are visible to the naked eye. ${ }^{3}$ The effect of our dental treatments means that these aerosols and splatter are widely dispersed through dental surgery. This is seen in the control agar plates with the largest CFU present on the dental arm, closest to where the procedure is taking place. The limitation of the alcohol products is the volatility resulting in a quick evaporation rate of the alcohol. The higher the alcohol percentage, the faster the associated evaporation rate. ${ }^{24}$ The rapid evaporation of the alcohols limit the surface contact time to achieve disinfection. The evaporation rate can be manipulated with the addition of various surfactants. ${ }^{19}$ Hence, the investigation for dental practices to possibly add a layer of decontamination. The use of a hydrogen peroxide fogger provides an opportunity for a "whole room" decontamination. Many manufactures of industrial-style "foggers" do not necessarily have a validation process that is repeatable by the end-user. This brings true efficacy into question. On average, a solo dentist could see five unique patients per day, ${ }^{25}$ but in most practices, the dentist treats more. The average time spent on each patient ranges from 15 to $45 \mathrm{~min}$. The average cleaning time between patients is $<5$ minutes.

The analysis indicated that alcohol spray, fogging, and the combination of fogging and alcohol spray had significantly fewer colonies than the control (Table 1).

The three disinfection methods presented a no-significant result for the number of colonies counted at the X-ray unit, right side desk, under the chair, dental arm, and left side desk. This result becomes apparent when dental surgery is considered 
where many items are present on the countertops. A limitation stated in the literature refers to the fogger being less effective for air-handling units and in situations where multiple objects are present in a room, requiring coverage by the hydrogen peroxide. ${ }^{26}$ The use of hydrogen peroxide foggers have shown the efficacy of decontaminating the air conditioners, ${ }^{27}$ but the influence on the dwell time and way the hydrogen peroxide fog settle on the various dental surgery surfaces could have played a role at the X-ray unit that was located directly under the air conditioner unit. The results from a controlled in vitro study indicated that inoculated discs located above and under tables were equally well disinfected. ${ }^{28}$ The agar plates under the dental chair were disinfected to a greater degree with the application of the hydrogen peroxide fogger when the SP of $13.9( \pm 20.4)$ CFU vs FG of $6.8( \pm 10.3)$ is considered. The use of $70 \%$ isopropanol without wiping is equally effective as spray and wipe. ${ }^{29}$ The SP group did reduce the CFU in all groups, but the SP + FG group performed the best. Surface cleaning with $70 \%$ alcohol, followed by fogging will provide the best result for surface decontamination in dental surgery.

Aerosol generation periods differ based on the complexity of the cavity design on the time required to complete a scale and polish with the ultrasonic scaler. It is important to note that the standard deviations for the various areas are large as the study was conducted based on varied treatment procedures performed in the general dental practice (Table 1). Additionally, the growth of bacteria and fungi was assessed on the agar plates, as these form the collective microbiological complement of dental surgery. The agar plates that were sprayed with alcohol and fogged had significantly less $(p<0.01)$ fungi than the agar plates that were only fogged. No damage was noted on any surface in the surgery as VHP under dehumidified conditions result in less damage than under condensation conditions. The effects of VHP had been studies under dehumidified conditions with 200 completed cycles minor changes of hardening/softening were noted for resins materials. Wood materials bleached and stainless steel discolored with zinc plated materials presenting with corrosion. The softening of nylon occurred while butyl rubber hardened. ${ }^{30}$

\section{Conclusion}

The disinfection of dental surgery requires sufficient time as it not only includes the working surfaces but also various inanimate objects. Surface disinfectant spray followed by hydrogen peroxide decontamination has potential for dental surgery, as the CFUs have been reduced further compared to spray alone and even just fog alone for all the various areas of the dental surgery that was assessed. Future directions of studies should not only evaluate the association between specific dental procedures and the CFU found on the agar plates but also the relationship of time spent on the procedure with the fast handpiece or ultrasonic scaler.

\section{Clinical Significance}

The most challenging clinical scenarios. The infection control protocols with hydrogen peroxide vapor as an adjunct to the $70 \%$ isopropanol alcohol spray would ensure the maximum efficacy of the disinfection protocols.

\section{Limitations}

There was no McFarland standard inoculation of the agar plates as a positive control since the study was a real-world data set compilation of aerosol that settle on agar plates in pre-determined areas, based on varied procedures that present during the day. The procedure performed in relation to the number of CFUs for that particular procedure in the various locations were not assessed. The other areas of a typical dental practice, e.g., reception and patient waiting areas were not assessed for CFU levels.

\section{References}

1. Weinstein RA, Hota B. Contamination, disinfection, and crosscolonization: are hospital surfaces reservoirs for nosocomial infection? Clin Infect Dis 2004;39(8):1182-1189. DOI: 10.1086/424667.

2. Singh A, Shiva Manjunath RG, Singla D, et al. Aerosol, a health hazard during ultrasonic scaling: a clinico-microbiological study. Indian J Dent Res 2016;27(2):160-162. DOI: 10.4103/0970-9290.183131.

3. Szymańska J. Dental bioaerosol as an occupational hazard in a dentist's workplace. Ann Agric Environ Med 2007;14(2):203-207. http://europepmc.org/abstract/MED/18247451.

4. Zemouri $C$, de Soet $H$, Crielaard W, et al. A scoping review on bio-aerosols in healthcare and the dental environment. PLoS One 2017;12(5):e0178007. DOI: 10.1371/journal.pone.0178007.

5. Peng X, Xu X, Li Y, et al. Transmission routes of 2019-nCoV and controls in dental practice. Int J Oral Sci 2020;12(1):1-6. DOI: 10.1038/s41368020-0075-9.

6. Weber DJ, Anderson D, Rutala WA. The role of the surface environment in healthcare-associated infections. Curr Opin Infect Dis 2013;26(4):338-344. DOI: 10.1097/QCO.0b013e3283630f04.

7. The South African Dental Association [Internet]. SADA dental clinical protocol in Response to COVID-19 Pandemic: A South African Private Practice Perspective. [Date accessed: 1 November, 2020]. Available from: https://cdn.sada.co.za/files/clinical-resources/downloads/ coronavirus/SADA Dental Clinical Protocols in Response to the Covid19 Pandemic 2020 A South African Perspective Final Draft 10-5-2020. pdf? $v=20200529142823$.

8. CDC, WHO [Internet]. Transmission-Based Precautions. [Date accessed: 1 November, 2020]. Available from: https://www.cdc.gov/ infectioncontrol/basics/transmission-based-precautions.html.

9. Veena HR, Mahantesha S, Joseph PA, et al. Dissemination of aerosol and splatter during ultrasonic scaling: a pilot study. J Infect Public Health 2015;8(3):260-265. DOI: 10.1016/j.jiph.2014.11.004.

10. Prospero E, Savini S. Microbial aerosol contamination of dental healthcare workers' faces and other surfaces in dental practice. Chic J 2013;24(2):139-141. DOI: http://www.jstor.org/stable/10.1086/502172.

11. Wells W. Aerodynamics of Droplet Nuclei. Cambridge, MA: Harvard University Press; 1955, pp. 1-423. https://www.cabdirect.org/ cabdirect/abstract/19562701497.

12. Mulder R, Layloo N, van Staden SM. COVID-19: focus on masks and respirators - implications for oral health-care workers. SADJ 2020;75(4):175-182. DOI: 10.17159/2519-0105/2020/v75no4a1.

13. Boyce JM. Alcohols as surface disinfectants in healthcare settings. Infect Control Hospital Epidemiol 2018;39(3):323-328. DOI: 10.1017/ ice.2017.301.

14. CDC-Center [Internet]. Guideline for Environmental Infection Control in Health-Care Facilities: Recommendations of CDC and the Healthcare Infection Control Practices Advisory Committee (HICPAC). [Date accessed 1 November, 2020]. Available from: http://www.cdc. gov/ncidod/hip/enviro/guide.htm.

15. Moorer WR. Antiviral activity of alcohol for surface disinfection. Int J Dent Hyg 2003;1(3):138-142. DOI: 10.1034/j.1601-5037.2003.00032.x.

16. Ratala W, Weber D, Guideline for Disinfection and Sterilization in Healthcare Facilities. [Internet]. 2008 [Date accessed 1 November, 2020]. Available at: https://www.cdc.gov/infectioncontrol/pdf/ guidelines/disinfection-guidelines.pdf.

17. Doerrbecker J, Friesland M, Ciesek S, et al. Inactivation and survival of hepatitis C virus on inanimate surfaces. J Infect Dis 2011;204(12):18301838. DOI: 10.1093/infdis/jir535. 
18. Rutala WA. APIC guideline for selection and use of disinfectants. Am J Infect Control 1996;24(4):313-342. DOI: 10.1016/s01966553(96)90066-8.

19. Rutala WA, Weber DJ. Selection of the ideal disinfectant. Infect Control Hosp Epidemiol 2014;35(7):855-865. DOI: 10.1086/676877.

20. Rutala WA, Weber DJ. Disinfection, sterilization, and antisepsis: an overview. Am J Infect Control 2019;47:A3-A9. DOI: 10.1016/j. ajic.2019.01.018.

21. McDonnell G, Russell AD. Antiseptics and disinfectants: activity, action, and resistance. Clin Microbiol Rev 1999;12(1):147-179. DOI: 10.1128/CMR.12.1.147.

22. Price PB. Reevaluation of ethyl alcohol as a germicide. Arch Surg 1950;60(3):492-502. DOI: 10.1001/archsurg.1950.01250010511006.

23. Block SS. Disinfection, Sterilization, and Preservation. Lippincott Williams \& Wilkins; 2001. https://books.google.co.za/books?id=3fkPJ17_TYC.

24. O'Hare KD, Spedding PL. Evaporation of a binary liquid mixture. Chem Eng J 1992;48(1):1-9. DOI: 10.1016/0300-9467(92)85001-P.

25. Gesko DS, Bailit HL. Dental group practice and the need for dentists. J Dent Educ 2017;81(8):eS120-eS125. DOI: 10.21815/ JDE.017.018.
26. Amaeze NJ, Shareef MU, Henriquez FL, et al. Influence of delivery system on the efficacy of low concentrations of hydrogen peroxide in the disinfection of common healthcare-associated infection pathogens. J Hosp Infect 2020;106(1):189-195. DOI: 10.1016/j. jhin.2020.06.031.

27. Taneja N, Biswal M, Kumar A, et al. Hydrogen peroxide vapour for decontaminating air-conditioning ducts and rooms of an emergency complex in northern India: time to move on. J Hosp Infect 2011;78(3):200-203. DOI: 10.1016/j.jhin.2011.02.013.

28. Wood JP, Calfee MW, Clayton M, et al. A simple decontamination approach using hydrogen peroxide vapour for bacillus anthracis spore inactivation. J Appl Microbiol 2016;121(6):1603-1615. DOI: 10.1111/jam.13284.

29. Graziano MU, Graziano KU, Pinto FMG, et al. Effectiveness of disinfection with alcohol $70 \%(\mathrm{w} / \mathrm{v})$ of contaminated surfaces not previously cleaned. Rev Lat Am Enfermagem 2013;21(2):618-623. DOI: 10.1590/S0104-11692013000200020.

30. Kimura T, Yahata H, Uchiyama Y. Examination of material compatibilities with ionized and vaporized hydrogen peroxide decontamination. J Am Assoc Lab Anim Sci 2020;59(6):703-711. DOI: 10.30802/AALAS-JAALAS-19-000165. 